\title{
Brain and Spinal MR Imaging Findings in Mucopolysaccharidoses: A Review
}

D.I. Zafeiriou and S.P. Batzios

\begin{abstract}
SUMMARY: MPS represents a group of rare hereditary disorders characterized by multisystem involvement due to intralysosomal GAG accumulation. Among various tissues, both the central and peripheral nervous system are affected in almost all types of the disease. Thus, brain and spinal MR imaging are valuable tools for the assessment of neurologic involvement, and there is evidence that they might be reliable markers demonstrating disease severity and efficacy of treatment options currently used in patients with MPS. We aimed to review the most prominent MR imaging features of patients with MPS, paying attention to the physiopathologic mechanisms responsible for these alterations. Along with the description of neuroimaging findings, existing data in relation to their correlation with the severity of neurologic involvement is discussed, while another topic of great importance is the effect of various therapeutic regimens in the progression of brain and spinal MR imaging alterations. Finally, recent data concerning MR spectroscopy studies in MPS are also critically discussed.
\end{abstract}

ABBREVIATIONS: ERT = enzyme replacement therapy; GAG = glycosaminoglycan; $\mathrm{HSCT}=$ hematopoietic stem cell transplantation; $\mathrm{MPS}=$ mucopolysaccharidoses; PVS = perivascular spaces; WMA = WM alterations

$\mathbf{M}^{\mathrm{B}}$ PS represents a heterogeneous group of inherited lysosomal storage disorders characterized by defective degradation of long-chain complex carbohydrates, called GAGs. ${ }^{1}$ To date, 11 distinct types of MPS have been described, each one as the result of deficient enzymatic activity of a specific lysosomal hydrolase. ${ }^{2}$ In each disease, the primary enzyme deficiency results in the accumulation of partially degraded GAGs within the lysosomes of various cell types, leading to progressive multisystem involvement. ${ }^{2}$ The clinical manifestations are diverse, including short stature and skeletal deformities, hepatosplenomegaly, hernias, and coarse facial features, whereas a different involvement of cardiovascular, respiratory, and CNS is observed in each type. ${ }^{1}$

In relation to neurologic involvement, symptoms vary widely, both in frequency and severity, among different types of MPS and individuals with each type of the disease. Intellectual impairment is a prominent feature in patients with MPS III and severe forms of MPS I, II, and VII, while normal cognition is retained in other types of the disease. ${ }^{1}$ The reason for this heterogeneity is not fully

From the First Department of Paediatrics, Aristotle University of Thessaloniki, Thessaloniki, Greece.

Please address correspondence to Dimitrios I. Zafeiriou, MD, PhD, First Department of Paediatrics, Aristotle University of Thessaloniki, Egnatia St 106, 54622,

Thessaloniki, Greece; e-mail address: jeff@med.auth.gr

--m Indicates open access to non-subscribers at www.ajnr.org

http://dx.doi.org/10.3174/ajnr.A2832 understood, especially because existing neuropathologic studies suggest neuron abnormalities even in patients in whom the CNS is not involved. ${ }^{3}$ Both the central and peripheral nervous system may be affected. The most common findings consistent with CNS involvement are mental retardation, hydrocephalus, progressive spasticity, seizures, cerebral infarction, ataxia, cervical cord compression and myelopathy, sleep apnea, optic atrophy, hearing impairment, and hyperactivity with aggressive behavior. ${ }^{4-6}$ Carpal and tarsal tunnel syndromes represent the most common features of peripheral nervous system impairment. ${ }^{6}$

The introduction of ERT along with potentially new therapeutic regimens aimed at the neurologic manifestations of MPS underlined the great need to identify reliable markers for predicting the outcome and monitoring CNS progression. Thus, neuroimaging studies are of great importance because CNS involvement has to be diagnosed early and carefully followed up to prevent serious complications.

Following a brief introduction about the findings in neuropathologic and CT neuroimaging studies, this article provides an overview of brain and spinal MR imaging features in patients with MPS. The existing literature in relation to the physiopathologic mechanisms leading to those specific deformities, the correlation of MR imaging features with the severity of neurologic involvement, and the effect of different treatment options at the progression of MR imaging findings is also discussed along with the description of neuroimaging findings. Finally, recent 
Overview of the most frequent brain and spinal MRI abnormalities according to disease type

\begin{tabular}{|c|c|c|c|c|c|}
\hline & $\begin{array}{c}\text { Signal } \\
\text { Alterations }\end{array}$ & $\begin{array}{l}\text { Enlarged } \\
\text { PVS }\end{array}$ & $\begin{array}{l}\text { Hydrocephalus/ } \\
\text { Ventriculomegaly }\end{array}$ & Atrophy & $\begin{array}{l}\text { Spinal } \\
\text { Stenosis }\end{array}$ \\
\hline \multicolumn{6}{|l|}{ MPS I } \\
\hline Hurler & +++ & +++ & +++ & ++ & ++ \\
\hline Hurler/Scheie & ++ & ++ & ++ & + & ++ \\
\hline Scheie & ++ & ++ & ++ & + & ++ \\
\hline MPS II & +++ & +++ & +++ & +++ & + \\
\hline \multicolumn{6}{|l|}{ MPS III } \\
\hline IIIA & ++ & ++ & ++ & + & + \\
\hline IIIB & ++ & ++ & + & +++ & - \\
\hline IIIC & - & - & - & - & - \\
\hline IIID & + & & \pm & + & - \\
\hline \multicolumn{6}{|l|}{ MPS IV } \\
\hline IVA & \pm & - & \pm & - & +++ \\
\hline IVB & \pm & - & - & - & +++ \\
\hline MPS VI & + & ++ & + & + & +++ \\
\hline MPS VII & ++ & - & - & - & + \\
\hline MPS IX & - & - & - & - & - \\
\hline
\end{tabular}

Note:- +++ indicates a constant finding; ++ , a frequent finding; + , a less frequent finding; \pm , rare; -, not described in the literature to our knowledge.

data concerning MR spectroscopic studies in MPS are also critically discussed.

\section{NEUROPATHOLOGIC AND CT NEUROIMAGING STUDIES}

CNS involvement in patients with MPS remains a critical issue. Neuropathologic studies with postmortem examinations revealed various brain and spinal deformities such as hydrocephalus, leptomeningeal thickening, and cavitary changes in the subcortical white matter with expanded, fluid-filled perivascular spaces, spinal stenosis, and cord compression. ${ }^{7}$ The first neuroradiologic studies in MPS were performed with the use of $\mathrm{x}$-ray CT and led to the identification of nonspecific imaging features, most prominent of which were the demonstration of low-attenuation white matter areas and the presence of ventricular and subarachnoid space dilation. ${ }^{8}$

The identification of white matter changes in the brain of patients with MPS led to the first MR imaging studies, because this imaging technique is undoubtedly the most sensitive method and represents, currently, the primary imaging technique for the detection of brain and spinal abnormalities in patients with MPS.

\section{THE MR IMAGING ERA}

Cranial and spinal MR imaging revealed a broad spectrum of changes in patients with MPS. The most prominent brain features, in almost all types of the disease, are white and gray matter changes, ventriculomegaly and hydrocephalus, cortical atrophy, and enlargement of the PVS, while in spinal neuroimaging, canal stenosis, cord compression, and myelopathy are usually present. The Table provides an overview of the most frequent MR imaging abnormalities according to disease type.

Although such findings have been described for $>30$ years, the evidence available was based mostly in case reports and small series of patients with MPS. Currently, it is widely accepted that MR imaging has significantly contributed to recent progress in the diagnostic work-up of these diseases and that it can be an extremely useful tool for monitoring the pathologic progression of patients with MPS.

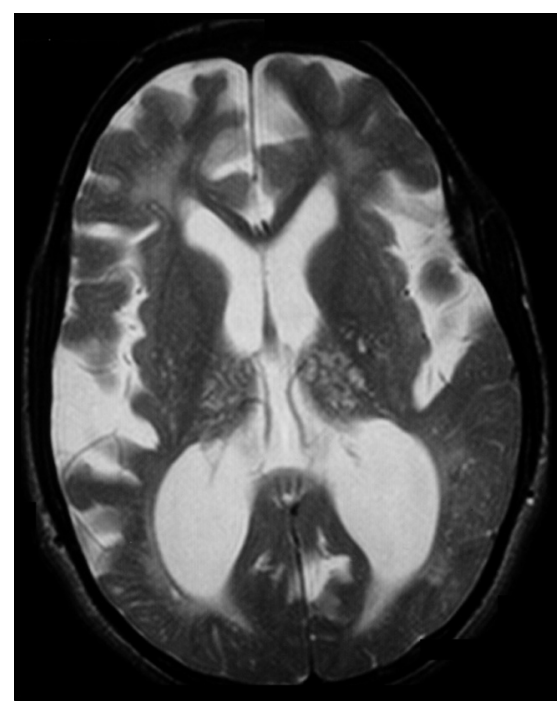

FIG 1. Brain T2-weighted axial MR image (TR/TE, 4000/99 ms) of a patient with MPS II at 21 years of age, demonstrating marked cortical atrophy and ventricular enlargement. Note also the extensive cribriform changes in both white matter and thalami.

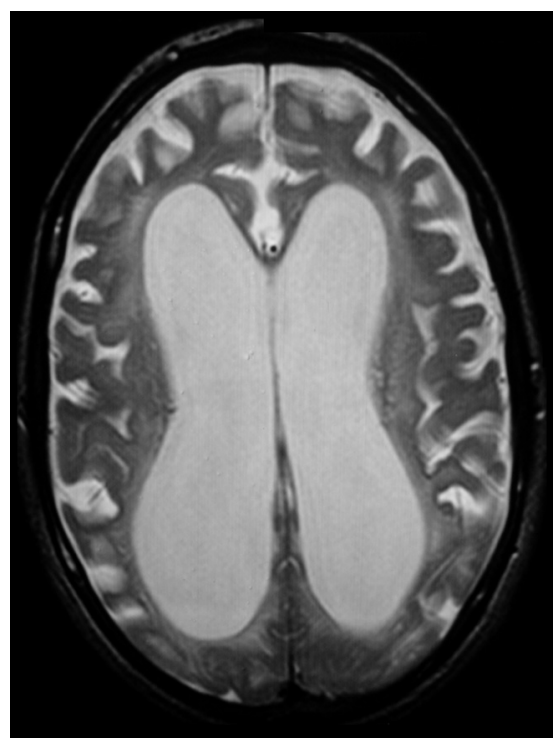

FIG 2. Brain axial T2-weighted image (TR/TE, $4000 / 99 \mathrm{~ms}$ ) of a 9-yearold patient with MPS II, demonstrating ventricular dilation and enlargement of the cortical sulci. WMA and disperse cystic lesions in the periventricular area are also noted.

\section{BRAIN MR IMAGING FINDINGS \\ White and Gray Matter Signal-Intensity Abnormalities}

Signal-intensity abnormalities in the brain parenchyma represent a constant neuroimaging feature (Figs 1-4). Nevertheless, the frequency, magnitude, and time of occurrence of WMA are variable in the different forms of MPS. ${ }^{9}$ Those changes are prominent in MPS I, ${ }^{10-19}$ II, ${ }^{12-14,16,18,20-27}$ IIIA, ${ }^{10,11}$ IIIB, ${ }^{18,21,28}$ IIID, ${ }^{29}$ and VII, ${ }^{13,19}$ whereas in MPS IV and VI, they are found to a lesser extent and may not become apparent until adulthood. ${ }^{16,19,21,30,31}$

WMA mainly consist of diffuse or focal areas of prolonged $\mathrm{T} 1$ and $\mathrm{T} 2$ relaxation times. These intensity changes are usually diffuse in patients with MPS III, while they are patchily distributed in other types of MPS. ${ }^{18}$ The most commonly affected region is the 


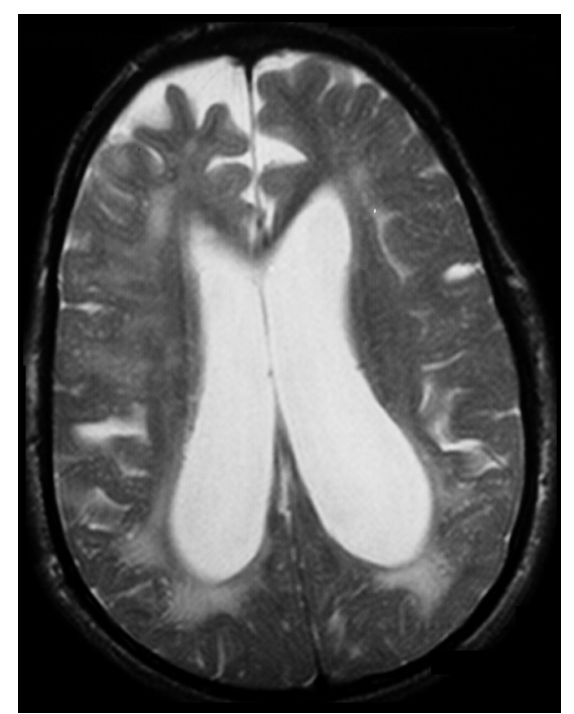

FIG 3. Brain T2-weighted axial MR image (TR/TE, $4000 / 99 \mathrm{~ms}$ ) of a patient with MPS II at 21 years of age, demonstrating WMA, mainly found in the retrotrigonal area of the brain, and cribriform changes in both periventricular and subcortical white matter. Note also the enlarged ventricles and cortical sulci in the frontal lobe.

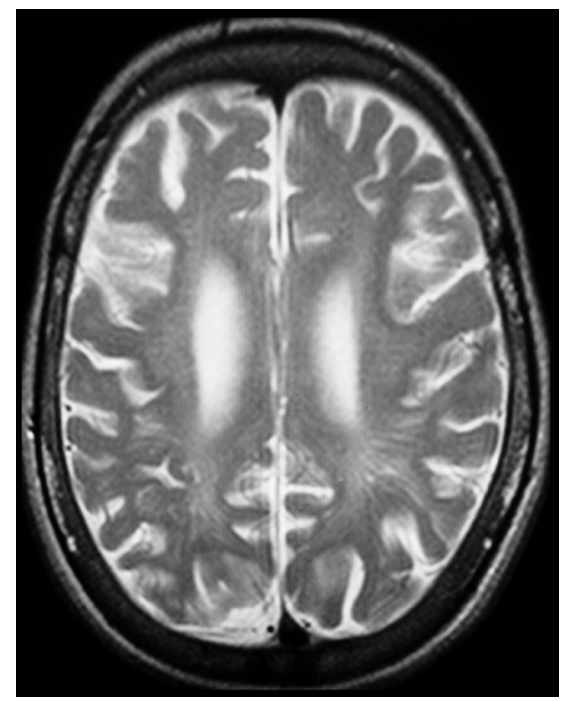

FIG 4. Brain T2-weighted axial MR image (TR/TE, $4000 / 99 \mathrm{~ms}$ ) of a patient with MPS IIIB at 9 years of age, demonstrating involvement of the white matter with signal-intensity alterations and PVS enlargement, cortical atrophy, and thickening of the diploe.

periventricular white matter, ${ }^{12,15}$ though signal-intensity abnormalities may appear in all parts of the cerebrum, affecting various brain lobes and subcortical regions. ${ }^{19,24}$ Concerning deep gray matter signal-intensity abnormalities, changes have been described in the region of the basal ganglia. ${ }^{16}$ As a result of T2weighted images showing abnormally high signal intensity, diminished contrast between the cerebral cortex and underlying white matter is usually demonstrated. ${ }^{16,27}$

A unique imaging feature in the brain of patients with MPS is the "honeycomb-like" appearance in the basal ganglia and thalami. It was first described by Shimoda-Matsubayashi et $\mathrm{al}^{25}$ in a 44-year-old patient with MPS II who exhibited patchy areas of increased and decreased signals in T1- and T2-weighted images in

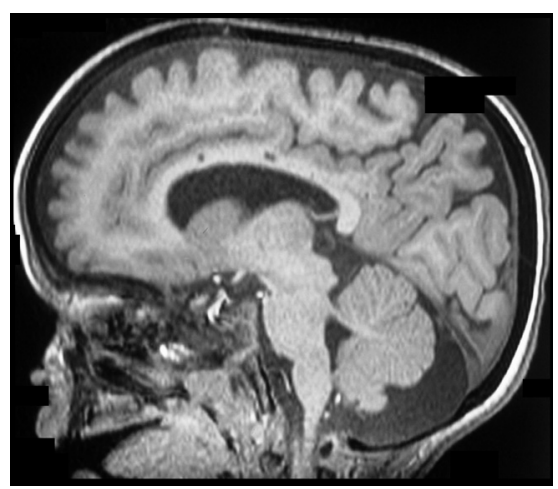

FIG 5. Brain sagittal TI-weighted image (TR/TE, 110/52 ms) of a patient with MPS II at 3 years of age. MR imaging demonstrates several cystlike dilated PVS in the body of the corpus callosum. A mega cisterna magna can be also observed.

the above-mentioned brain regions. Apart from MPS II, this finding was also described in patients with MPS I and IIIB. ${ }^{12,14,15,20,25,28}$

The pathophysiologic mechanisms causing MR imaging signal-intensity alterations are not fully understood. Nevertheless, diffuse WMA and "honeycomb-like" appearance might be indicative of a process that leads to changes characterized by the accumulation of GAGs and gangliosides in neurons and astrocytes, ${ }^{16,18,21,32,33}$ leading to neuronal loss, gliosis, delayed myelination, or/and demyelination. ${ }^{9,10,18,21,26,32}$ Among other theories, increase of fluid content in the $\mathrm{PVS}^{7}$ and lacunar infarctions have been proposed. ${ }^{10,21}$

\section{Brain Lesions and Enlarged PVS}

One of the most common abnormalities found in patients with MPS are the cystic lesions that correspond to enlargement of VirchowRobin PVS (Figs 1, 2, 4, and 5). These sharply defined foci demonstrate a high signal intensity, isointense to CSF on T2-weighted and FLAIR images, and low signal intensity on T1-weighted images, which result in the so- called "cribriform" or "spindle-like" MR imaging changes. ${ }^{23,34}$ Although prominent in most patients with MPS, this feature is nonspecific because it was found in numerous pathologic conditions and healthy subjects as well. ${ }^{35}$ Enlarged PVS have been described so far in MPS $\mathrm{I}^{10-13,15,17-19,36}$ II, ${ }^{10,12,13,18,20-23,27,37}$ IIIA, ${ }^{10,38,39}$ IIIB, ${ }^{28,40,41}$ and VI. ${ }^{16,18,19,30}$ In general, children with MPS I and II show the most profound enlargement of PVS, giving rise to a "sieve-like" appearance. ${ }^{18} \mathrm{In}$ relation to their localization, cystic lesions tend to be linear, varying from patchy to diffuse, ${ }^{12}$ and affect most brain parts, with periventricular white matter being the most common, followed by the corpus callosum (Fig 5), basal ganglia, subcortical white matter, thalami (Fig 4), and brain stem. ${ }^{11,12,15,18,20,27,28,37,38,42}$ In most, dilated PVS range from 2 to $8 \mathrm{~mm}$ in diameter, ${ }^{12,32}$ though giant cavitary lesions of $1.5 \mathrm{~cm}$ in the brain MR imaging of 1 patient with Hurler-Scheie syndrome were also described in the literature. $^{17}$

The cystic areas on MR imaging most likely correspond to perivascular expanded fluid spaces, first described as a neuropathologic autopsy sign by Dekaban and Constantopoulos. ${ }^{7}$ The presence of cribriform changes as one of the earliest lesions of the white matter in young patients mostly led to the notion that these 
might be present only for a short time, followed by WMA and atrophy; thus, these are possibly related to the rate and specificity of glycolipid storage in the neurons. ${ }^{10,28}$ Present data do not clarify the physiopathologic mechanisms responsible for the formation of cystic lesions, and there are currently 2 main explanatory theories. Parsons et $\mathrm{al}^{20}$ first hypothesized that these areas are formed due to GAG storage around the vessels. This notion is supported by the fact that enlarged PVS were not present in all patients with MPS, though GAG storage was always present, regardless of disease type. ${ }^{38}$ Almost 10 years later, Matheus et $\mathrm{al}^{12}$ hypothesized that PVS dilation may reflect impairment of CSF reabsorption due to mucopolysaccharide deposits in the leptomeninges. This theory is currently gaining ground, and it is nowadays believed that enlarged PVS might represent a sensitive biomarker showing abnormal CSF circulation.

\section{Hydrocephalus and Ventricular Enlargement}

Communicating hydrocephalus is a well-recognized feature that occurs in most MPS types. ${ }^{15-18,22,26,28,43-45}$ It is usually only slowly progressive, with enlargement of subarachnoid and ventricular spaces representing its main neuroradiologic findings. In general, ventricular enlargement is considered a common feature in MPS (Figs 1-3). MPS I ${ }^{10-19,21,36,42,43}$ and $\mathrm{II}^{10,12,13,15,16,18-20,22,24}$ show marked ventriculomegaly, whereas in types IIIA, ${ }^{19,38,39,45}$ IIIB, ${ }^{18,19,28}$ IIID, ${ }^{29}$ IVA, ${ }^{15,16}$ and VI, ${ }^{16,19,30,44}$ ventricle enlargement is less frequent. Manara et $\mathrm{al}^{22}$ recently showed that all patients with Hunter syndrome in their study had ventriculomegaly. The third and lateral ventricles are usually involved, ${ }^{12}$ though there are cases in which enlargement of the entire ventricular system was observed. ${ }^{17}$ In relation to the origin of ventriculomegaly, most authors agree that it is the combined result of cerebral atrophy secondary to neurologic degeneration, venous hypertension, and defective CSF reabsorption. ${ }^{12,26}$ In the case of hydrocephalus, most authors agree that the deposition of storage material in the meninges may impair the function of pacchionian granulations and lead to abnormal CSF reabsorption and finally communicating hydrocephalus. ${ }^{15,34}$ Stöckler et $\mathrm{al}^{43}$ described the case of a 19-month-old patient with Hurler-Scheie syndrome who developed high-pressure hydrocephalus as a complication of an intracranial tumor. To our knowledge, this is the only report of a brain tumor in a patient with MPS.

In addition to ventriculomegaly, enlarged subarachnoid spaces represent a prominent feature of most MPS types, especially I and II. ${ }^{11,12,38}$ These spaces are usually found in the sella turcica, middle cranial, and posterior fossa, and around the optic nerves; because they are combined with ventricle enlargement, abnormal CSF reabsorption is considered to be the causative physiopathologic mechanism. ${ }^{12}$

\section{Atrophic Changes}

Differentiation between communicating hydrocephalus and brain atrophy is a demanding task because both conditions share common clinical and neuroradiologic features (enlarged ventricular and subarachnoid spaces). This is actually the reason that some authors merged data regarding those 2 situations and considered them as one. ${ }^{22}$ Nevertheless, Lee et $\mathrm{al}^{10}$ defined cerebral atrophy as the presence of dilated brain sulci. It represents one of the most prevalent MR imaging features in patients with MPS II $^{12,18,20-22,24,26}$ and IIIB, ${ }^{18,21,28,40,41}$ while it is also described in MPS I, ${ }^{11,12,21,43}$ IIIA, ${ }^{39,45}$ IIID, ${ }^{29}$ and VI. ${ }^{21}$ Cerebral atrophy is predominantly cortical and diffuse ${ }^{18}$ (Figs 1-4) and may be symmetric or asymmetric. ${ }^{12,26}$ In relation to the asymmetry, no explanation is currently valid. Matheus et $\mathrm{al}^{12}$ hypothesized that it may be the result of irregular GAG storage or reabsorption of the CSF. The pathophysiology of brain atrophy remains unclear. It is probably the result of neuronal death and gliosis induced by the accumulation of mucopolysaccharides, ${ }^{10}$ though other theories such as microglial activation, enlargement of CSF spaces, ectopic dendritogenesis, and neuroaxonal dystrophy have been proposed. ${ }^{20,46,47}$

Apart from cerebral atrophy, corpus callosum thinning was also described in patients with MPS I, ${ }^{19}$ II, ${ }^{19}$ IIIA, ${ }^{38,39}$ IIIB, ${ }^{28,41}$ and VI, ${ }^{19}$ most probably as a result of ventricular dilation and enlarged PVS within this brain structure. Finally, cerebellar atrophy was found in 2 patients with MPS I ${ }^{14}$ and IIIB. ${ }^{40}$

\section{Posterior Fossa Abnormalities}

Patients with MPS have also been described as having abnormal MR imaging features concerning the area of the posterior cranial fossa. The most common among these findings is the presence of a mega cisterna magna (Fig 5), which is frequent in patients with MPS II ${ }^{18,20,22,27}$ and has been also reported in MPS I and IIIB. ${ }^{18}$ Lee et $\mathrm{al}^{10}$ first described, in their cohort of patients, intracranial arachnoid cysts. Posterior fossa arachnoid cysts and mega cisterna magna represent CSF-filled spaces of unknown etiology, though splitting or duplication of the arachnoid matter, brain structure agenesis, and disturbances of CSF circulation represent the most predominant explanatory theories. ${ }^{48,49}$ A small posterior fossa has been described in MPS II. ${ }^{22}$

Concerning the cerebellum, white matter changes were demonstrated in MPS IIIB, ${ }^{28}$ and I, ${ }^{17}$ while finally, Chiari I malformation was reported in patients with MPS II (Fig 6). ${ }^{22}$

\section{Other Brain MR Imaging Findings}

Apart from the above-mentioned abnormalities, there are also case descriptions of less frequent or unique MR imaging features. One finding described in several patients with MPS is the presence of pituitary sella abnormalities, found in MPS I, ${ }^{17,42}$ II, ${ }^{18,20,22}$ IIIA, ${ }^{38,45}$ IV, ${ }^{50}$ and VI. ${ }^{16,31}$ Different variations have been demonstrated with the sella being small ${ }^{38}$ or enlarged, ${ }^{17}$ with ski-shoe, ${ }^{42} \mathrm{~J},{ }^{20,22,31}$ or omega shape, ${ }^{17,22}$ while frequently found was an empty sella appearance. ${ }^{18,31,50}$ Those abnormalities have been associated with growth hormone deficiency. ${ }^{31}$ Although the cause of pituitary sella abnormalities is not yet established, Wilson et $\mathrm{al}^{51}$ have demonstrated a dysfunction of bone maturation in an MPS animal model, which may account for the above-mentioned skull deformities. Moreover, an enlarged pituitary gland was found in a child with MPS IIIA who had precocious puberty. ${ }^{45}$

Another interesting MR imaging study was performed in a child with MPS I. ${ }^{11}$ Barone et $\mathrm{al}^{11}$ described the case of a patient with Hurler-Scheie syndrome who had WMA that gradually progressed and affected the U-fibers, giving rise to a pattern that is typical of leukodystrophies. Finally, other MR imaging features described in various case reports and small case series are extreme 


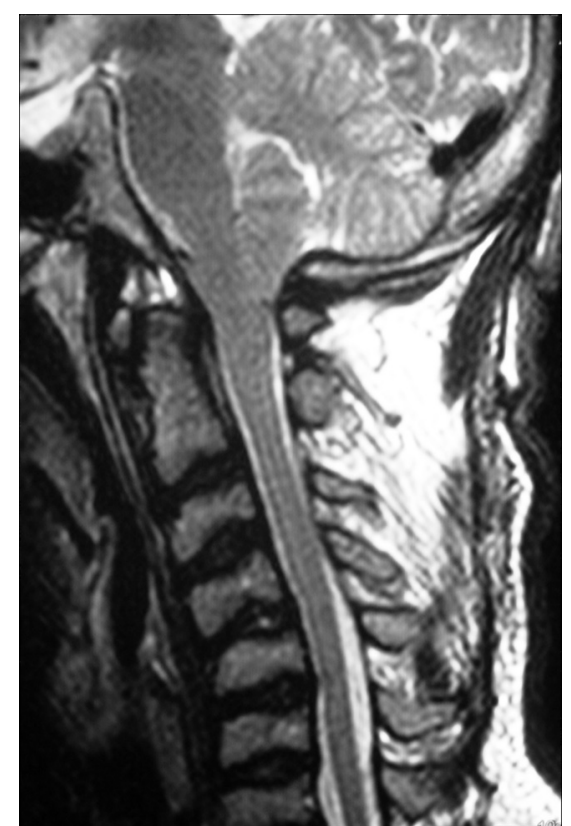

FIG 6. Spinal sagittal T2-weighted image (TR/TE, 3350/115 ms) of a patient with MPS VI at 25 years of age. MR imaging demonstrates canal stenosis, vertebral abnormalities, and a mild Chiari I malformation.

dilation of the venous sinuses and thickening of the diploe in MPS IIIB (Fig 4), ${ }^{28}$ optic canal stenosis and optic nerve atrophy in MPS $\mathrm{VI},{ }^{44}$ unilateral subdural hematoma in MPS $\mathrm{II}^{37}$ and IIIB due to cerebral vasculopathy, ${ }^{52}$ and pseudotumor cerebri in MPS IV, as demonstrated by the elevated intracranial pressure with normalsized ventricles. ${ }^{53}$

\section{SPINAL MR IMAGING FINDINGS}

\section{Craniocervical Junction Abnormalities}

Spinal abnormalities account for a high portion of neurologic manifestations, especially in the types of the disease in which the CNS is not involved and patients maintain a normal intellect. Such manifestations are exercise intolerance, limb weakness, apnea, spastic quadriparesis, and hemiparesis, and sudden death can usually occur following minor trauma. ${ }^{15,18,54}$ The most common craniocervical junction MR imaging features are spinal stenosis, compressive myelopathy, odontoid dysplasia, atlantoaxial instability, and dural thickening.

Compromise of the central canal and spinal cord (Fig 6) with or without signs of compressive myelopathy is not an uncommon feature in patients with MPS and may even be the first sign leading to disease diagnosis. ${ }^{55}$ It represents a prominent finding in MPS IV, ${ }^{11,16,18,50,54,56,57} \mathrm{VI},{ }^{16,18,19,30,44}$ and $\mathrm{I},{ }^{11,15,16,18,19,58-60}$ and cases of MPS II, ${ }^{19,22,55,61}$ IIIA, ${ }^{19}$ and VII ${ }^{18,62}$ have also been described. MR images show abnormal signal intensity at the level of myelopathy, usually in the cervical area, and the thoracolumbar region can be also affected. ${ }^{59}$ Mut et al ${ }^{63}$ described the case of an 18-year-old male patient with Maroteaux-Lamy syndrome who exhibited multilevel myelopathy affecting both cervical and thoracolumbar spinal regions. Spinal cord compression is believed to be multifactorial, but there are 2 main proposed physiopathologic mechanisms. The first is related to GAG deposition and infiltra- tion of surrounding tissues and especially the dura mater by a soft-tissue mass. ${ }^{15,18,30,44,59}$ This mass is typically iso- or hypointense on T1- and hypointense on T2-weighted images. MPS IV, VI, and I show a high propensity for dural thickening, while this finding has also been described to a lesser extent in patients with MPS II. ${ }^{16,55,61}$ The second mechanism proposes vertebral anomalies leading to deformities and atlantoaxial instability due to dysfunction of the odontoid process as etiopathologic mechanisms for cord compression..$^{50,55,57,61,63,64}$ Odontoid abnormalities can vary from complete aplasia ${ }^{56}$ to varying degrees of hypoplasia, and have been described mostly in patients with MPS IV, VI, and I. ${ }^{16,30,58,60,63}$ Using MR imaging to screen patients with MPS for evidence of spinal cord compression is critical and can be very helpful in optimizing the time for surgical intervention because those patients have significant problems related to intubation and show high rates of mortality due to upper respiratory passage abnormalities.

Apart from the above-mentioned spinal MR imaging abnormalities, Hite et $\mathrm{al}^{65}$ described the case of a patient with Maroteaux-Lamy who developed holocord syringomyelia; in another case description, signal-intensity changes within the cervical cord were demonstrated, though the authors were not able to clarify whether this finding represented sites of GAG storage or demyelination. ${ }^{20}$ Finally, Samoa et $\mathrm{al}^{66}$ described the case of a 10-year-old patient with MPS IIIB who demonstrated hemiatrophy of the spinal cord.

\section{Vertebral and Disk Abnormalities}

One of the most common manifestations in MPS is dysostosis multiplex, and patients exhibit various degrees of bone and joint involvement, mostly due to GAG deposition and dysfunction of bone maturation. ${ }^{51}$ Thus, spinal MR imaging demonstrates various abnormalities concerning the vertebral bodies and intersomatic disks (Fig 6). Characteristic features are platyspondylia, and bullet shape and beaking of vertebrae, while disks were described as dehydrated. ${ }^{15,20}$

\section{MR SPECTROSCOPY}

MR spectroscopy represents an objective diagnostic tool, which determines the concentration of brain metabolites and is able to provide possible correlations between those compounds and the underlying neuronal damage. It has recently become an additional tool in the evaluation of neurologic involvement in patients with MPS, and numerous studies have proposed the use of MR spectroscopy metabolite alterations as biomarkers demonstrating disease severity. A decade ago, Takahashi et al, ${ }^{13}$ by using MR spectroscopy, found a resonance that was higher than that of myo-inositol and correlated this finding with in vitro GAG accumulation. Additionally, they have shown an elevation in the choline/creatine ratio in white matter lesions, speculating that this finding was the result of myelin damage, increased glial proliferation, or elevated cell membrane synthesis. ${ }^{13}$ An important issue addressed in their work, as well as in the experiments of Vedolin et $\mathrm{al},{ }^{26}$ was whether MR spectroscopic alterations could represent a possible biomarker of cognitive impairment in patients with MPS. The latter research group has found that cognitively impaired patients demonstrate a higher myo-inositol/creatine ratio, 
hypothesizing that this MR spectroscopic finding is due to GAG accumulation, increased astrocyte cell volume, or altered glial metabolism. $^{26}$

Their data have been confirmed and further extended by Davison et $\mathrm{al}^{67}$ in a cohort of patients with MPS II. Decreased $N$ acetylaspartate, total choline and glutamate in the white matter, and an elevation of myo-inositol were the main findings. Additionally, they have shown an alteration in $\mathrm{N}$-acetylaspartate concentration with disease duration, providing evidence that MR spectroscopy could be used as a monitoring tool demonstrating progressive neurologic impairment. ${ }^{67}$ Nevertheless, these data were not confirmed in another study, in which metabolite ratios examined by MR spectroscopy did not show any correlation with disease duration, age of patients, and biochemical findings of urinary GAG excretion and enzymatic activity. ${ }^{68}$ In summary, all the above data demonstrate that MR spectroscopy could represent an adjunct to MR imaging in the evaluation of neurologic involvement, though more confirming studies are needed.

\section{ADDITIONAL MR IMAGING TECHNIQUES}

Apart from conventional techniques, advanced MR imaging approaches such as DTI have been used recently, and there is an early experience that should be mentioned. Those images usually demonstrate iso- or faint hyposignal within the white matter, which represents sites of dysmyelination and low-grade demyelination, with no sites of hypersignal, which could suggest myelin edema. ${ }^{9}$ Diffusion coefficient and anisotropy remained within the normal range, and this method might be proved of great importance in the differentiation among white matter lesions observed in various diseases. ${ }^{69}$ Sener ${ }^{70}$ has recently studied DTI patterns in 34 patients with various metabolic and toxic brain disorders. Among them, there were 2 patients with MPS who showed an elevated diffusion pattern, suggesting that this finding corresponds to disintegration of the brain tissue.

To the best of our knowledge, no studies concerning functional MR imaging findings in patients with MPS have been reported.

\section{CLINICAL ASPECTS OF MR IMAGING FINDINGS IN MPS}

A critical issue of brain and spinal MR imaging findings in patients with MPS is whether they keep up with the pace of both phenotype and disease progression. Several studies deal with the correlation of neuroimaging features with the presence of cognitive impairment; however, the relationship between these is rather controversial. Many researchers came to the conclusion that the presence and extension of imaging abnormalities do not correspond to the severity of mental retardation. This lack of correlation was consistent for WMA, ${ }^{11,12,14,20,21,23,25,38,41}$ enlargement of the PVS, ${ }^{10,12,18,23}$ cerebral atrophy, ${ }^{12,26,41}$ and ventriculomegaly. ${ }^{12,26}$ On the other hand, a significant number of studies did find more severe neuroimaging abnormalities in patients with impaired cognitive profiles. ${ }^{10,15,19,21,26}$ In most of these studies, differences were usually attributed to the inclusion of patients of various MPS types, which were regarded as a unique study group, and the differences could probably be resolved with the use of a more detailed scoring tool. Fan et $\mathrm{al}^{37}$ were the first to use quantitative volumetric methods to differentiate MPS II patients with and without mental retardation. They found that patients with cognitive impairment exhibit decreased brain tissue and increased lateral ventricle size expressed by a fraction of the intracranial volume. Recent data have shown a significant correlation between the extent of WMA, third ventricle enlargement, and occipital hyperostosis and the presence of mental retardation, whereas a mega cisterna magna was associated with the attenuated phenotype of patients with MPS II. ${ }^{22}$

In relation to the effect of disease progression on brain and spinal MR imaging abnormalities, several authors have demonstrated that the most common neuroimaging features, such as WMA, atrophy, ventricular enlargement, honeycomb-like appearance, and spinal stenosis follow a trend of worsening with increasing age, ${ }^{14,20,22,25,68}$ whereas others described the same features as age-independent factors, showing no correlation with disease duration. ${ }^{12,19,21}$ Aiming to investigate the influence of aging on MR imaging findings, Vedolin et $\mathrm{al}^{68}$ used automated and semiautomated segmentation tools in the study of MR imaging findings in a large cohort of patients with MPS, measuring the normalized cerebral volume, CSF volume, ventricular volume, and lesion load. They found that patients with longer disease duration exhibit more pronounced white matter lesions and excessive atrophy. Also, they tried to correlate neuroimaging findings with biochemical markers of the disease, such as enzyme levels and urinary GAG excretion, but no association was found. Finally, Manara et $\mathrm{al}^{22}$ have shown a positive correlation between age and dural thickening, while sella deformities and odontoid hypoplasia were inversely related to disease duration.

A matter of great significance is whether the presence of described abnormalities correlates with the existence of other MR imaging features. This is really important because possible associations could exhibit common physiopathologic mechanisms or, on the other hand, an independent course of neuroimaging findings. Lee et $\mathrm{al}^{10}$ first observed that cribriform changes were inversely correlated with the gravity of WMA, cerebral atrophy, and ventriculomegaly. In relation to WMA, Murata et $\mathrm{al}^{21}$ have shown that this feature is related to PVS enlargement. Finally, present data demonstrated that the occurrence of a mega cisterna magna does not correlate with the presence of enlarged ventricles and subarachnoid spaces, whereas regarding spinal deformities, no association could be proved between the severity of odontoid hypoplasia and the presence of canal stenosis or dura thickening. ${ }^{22}$

\section{EFFECT OF THERAPEUTIC REGIMENS IN MR IMAGING FINDINGS}

The management of MPS is really challenging, and currently there are few therapeutic options aiming to correct the enzyme deficiency. Among these, HSCT and ERT represent the most commonly used, and existing data show a controversial impact of those treatment modalities on brain and spinal MR imaging findings.

In relation to HSCT, there have been studies showing a significant improvement of brain MR imaging features with regression of lesions and WMA following successful engraftment. ${ }^{13,18,42}$ Those alterations were also accompanied by favorable neurologic outcome. ${ }^{36}$ In addition, although it is widely accepted that HSCT has limited or no effect on skeletal manifestations of the disease, 
current research has shown that odontoid dysplasia can be normalized with the use of this treatment technique. ${ }^{58,60,71}$ On the other hand, there are existing data showing that the efficacy of this treatment option is insufficient for both brain and spinal MR imaging features, with progression of neuroimaging findings with time. $^{59}$

ERT is currently available for MPS I, II, and VI and has been shown to improve cardinal signs of the disease, such as hepatosplenomegaly, urinary GAG excretion, respiratory function, and joint and cardiovascular involvement. Nevertheless, because the nervous system, due to the existence of the BBB, is considered "impermeable" to the intravenous administration of enzymes, ERT is not expected to improve neurologic manifestations. Yet, limited data show that even the CNS seems to be improved after the administration of ERT. Wang et $\mathrm{al}^{72}$ first showed that ERT can reverse or stabilize neuroimaging findings, and they raised important questions in relation to the efficacy of this treatment option on the CNS. The same authors proposed, as possible mechanisms for the improvement of MR imaging features, the correction of CSF dynamics, the repair and reduction of GAG accumulation through the $\mathrm{BBB}$, the suppression of inflammation, and finally the possible penetrance of the BBB from the administered enzyme, which reverses MR imaging features but not neurologic symptoms. ${ }^{72}$ More researchers further supported the same notion because they demonstrated improvement of neuroradiologic features. ${ }^{36}$ Nevertheless, other studies have shown a worsening of brain and spinal MR imaging findings despite ERT introduction, implying that neurologic MR imaging alterations, even at the level of structures without the $\mathrm{BBB}$, are difficult to reverse once they have occurred. ${ }^{22,68}$

As an alternative to direct enzymes into the brain and reverse neuroimaging features, the application of ERT via intrathecal administration was used in 2 patients with MPS I ${ }^{73}$ and VI. ${ }^{74}$ Follow-up MR imaging studies showed no worsening of WMA and spinal cord compression in the first patient; yet, intrathecal administration of the enzyme in the second patient led to generalized hypotonia, probably due to reduction of GAG storage and revelation of the instability of the cervical vertebrae.

The above data underline the supposition that MR imaging could probably represent a valuable tool in the assessment of the efficacy of various treatment options and could provide reliable markers to estimate neurologic disease burden.

\section{CONCLUSIONS}

Brain and spinal MR imaging represent important tools in the diagnostic work-up of MPS. The demonstration of signal-intensity abnormalities, enlargement of the PVS, ventriculomegaly, atrophy, and cord compression is a prominent feature in almost all types of the disease. Nevertheless, those abnormalities are nonspecific and have been described in numerous neurologic conditions, static or degenerative. Thus, more systematic studies are needed to evaluate thoroughly the spectrum of MR imaging findings and to correlate these with currently used clinical and biochemical markers of MPS. This could also help in delineating the nature of the described alterations, a matter of great debate, and finally in highlighting the use of MR imaging as a marker of great predictive value for neurologic outcome, thus enabling a better understanding of the etiopathologic mechanisms of this complex group of syndromes. Accordingly, the application of a MR imaging protocol for the evaluation of the progress of brain and spinal abnormalities by all health care professionals in the same manner could be of extreme importance in monitoring the effect of different therapeutic regimens.

\section{REFERENCES}

1. Neufeld EF, Muenzer J. The Mucopolysaccharidoses. In: Scriver CR, Beaudet AL, Sly WS, eds. The Metabolic and Molecular Bases of Inherited Disease. 8th ed. New York: McGraw-Hill; 2001;3421-52

2. Clarke LA. The mucopolysaccharidoses: a success of molecular medicine. Expert Rev Mol Med 2008;10:e1

3. Gilles FH, Deuel RK. Neuronal cytoplasmic globules in the brain in Morquio's syndrome. Arch Neurol 1971;25:393-403

4. Muenzer J. The mucopolysaccharidoses: a heterogeneous group of disorders with variable pediatric presentations. J Pediatr 2004;144: 27-34

5. Wraith JE. The mucopolysaccharidoses: a clinical review and guide to management. Arch Dis Child 1995;72:263-67

6. Al Sawaf S, Mayatepek E, Hoffmann B. Neurological findings in Hunter disease: pathology and possible therapeutic effects reviewed. J Inherit Metab Dis 2008;31:473-80

7. Dekaban AS, Constantopoulos G. Mucopolysaccharidosis type I, II, IIIA and V: pathological and biochemical abnormalities in the neural and mesenchymal elements of the brain. Acta Neuropathol 1977;39:1-7

8. Nelson J, Grebbell FS. The value of computed tomography in patients with mucopolysaccharidosis. Neuroradiology 1987;29:544-49

9. Patay Z. Diffusion-weighted MR imaging in leukodystrophies. Eur Radiol 2005; 15:2284-303

10. Lee C, Dineen TE, Brack M, et al. The mucopolysaccharidoses: characterization by cranial MR imaging. AJNR Am J Neuroradiol 1993;14:1285-92

11. Barone R, Parano E, Trifiletti RR, et al. White matter changes mimicking a leukodystrophy in a patient with mucopolysaccharidosis: characterization by MRI. J Neurol Sci 2002;195:171-75

12. Matheus MG, Castillo M, Smith JK, et al. Brain MRI findings in patients with mucopolysaccharidosis types I and II and mild clinical presentation. Neuroradiology 2004;46:666-72

13. Takahashi Y, Sukegawa K, Aoki M, et al. Evaluation of accumulated mucopolysaccharides in the brain of patients with mucopolysaccharidoses by (1) $\mathrm{H}$ magnetic resonance spectroscopy before and after bone marrow transplantation. Pediatr Res 2001;49:349-55

14. Gabrielli O, Salvolini U, Maricotti M, et al. Cerebral MRI in two brothers with mucopolysaccharidosis type I and different clinical phenotypes. Neuroradiology 1992;34:313-15

15. Kulkarni MV, Williams JC, Yeakley JW, et al. Magnetic resonance imaging in the diagnosis of the cranio-cervical manifestations of the mucopolysaccharidoses. Magn Reson Imaging 1987;5:317-23

16. Taccone A, Tortori Donati P, Marzoli A, et al. Mucopolysaccharidosis: thickening of dura mater at the craniocervical junction and other CT/MRI findings. Pediatr Radiol 1993;23:349-52

17. Rauch RA, Friloux LA 3rd, Lott IT. MR imaging of cavitary lesions in the brain with Hurler/Scheie. AJNR Am J Neuroradiol 1989;10:1-3

18. Seto T, Kono K, Morimoto K, et al. Brain magnetic resonance imaging in 23 patients with mucopolysaccharidoses and the effect of bone marrow transplantation. Ann Neurol 2001;50:79-92

19. Gabrielli O, Polonara G, Regnicolo L, et al. Correlation between cerebral MRI abnormalities and mental retardation in patients with mucopolysaccharidoses. Am J Med Genet A 2004;125A:224-31

20. Parsons VJ, Hughes DG, Wraith JE. Magnetic resonance imaging of the brain, neck and cervical spine in mild Hunter's syndrome (mucopolysaccharidoses type II). Clin Radiol 1996;51:719-23

21. Murata R, Nakajima S, Tanaka A, et al. MR imaging of the brain in patients with mucopolysaccharidosis. AJNR Am J Neuroradiol 1989;10:1165-70 
22. Manara R, Priante E, Grimaldi M, et al. Brain and spine MRI features of Hunter disease: frequency, natural evolution and response to therapy. J Inherit Metab Dis 2011;34:763-80. Epub 2011 Apr 5

23. Shinomiya N, Nagayama T, Fujioka Y, et al. MRI in the mild type of mucopolysaccharidosis II (Hunter's syndrome). Neuroradiology 1996;38:483-85

24. Finn CT, Vedolin L, Schwartz IV, et al. Magnetic resonance imaging findings in Hunter syndrome. Acta Paediatr Suppl 2008;97:61-68

25. Shimoda-Matsubayashi S, Kuru Y, Sumie H, et al. MRI findings in the mild type of mucopolysaccharidosis II (Hunter's syndrome). Neuroradiology 1990;32:328-30

26. Vedolin L, Schwartz IV, Komlos M, et al. Correlation of MR imaging and MR spectroscopy findings with cognitive impairment in mucopolysaccharidosis II. AJNR Am J Neuroradiol 2007;28:1029-33

27. Zafeiriou D, Auggoustidou-Savvopoulou PA, Papadopoulou FA, et al. Magnetic resonance imaging findings in mild mucopolysaccharidosis II (Hunter's syndrome). Eur J Paediatr Neurol 1998;2:153-56

28. Zafeiriou DI, Savvopoulou-Augoustidou PA, Sewell A, et al. Serial magnetic resonance imaging findings in mucopolysaccharidosis IIIB (Sanfilippo's syndrome B). Brain Dev 2001;23:385-89

29. Ozand PT, Thompson JN, Gascon GG, et al. Sanfilippo type D presenting with acquired language disorder but without features of mucopolysaccharidosis. J Child Neurol 1994;9:408-11

30. Thorne JA, Javadpour M, Hughes DG, et al. Craniovertebral abnormalities in type VI mucopolysaccharidosis (Maroteaux-Lamy syndrome). Neurosurgery 2001;48:849-52

31. Büyükgebiz B, Eroğlu Y, Kovanlikaya I, et al. Maroteaux-Lamy syndrome associated with growth hormone deficiency. J Pediatr Endocrinol Metab 1995;8:305-07

32. Johnson MA, Desai S, Hugh-Jones K, et al. Magnetic resonance imaging of the brain in Hurler syndrome. AJNR Am J Neuroradiol 1984;5:816-19

33. Walkley SU, Thrall MA, Haskings ME, et al. Abnormal neuronal metabolism and storage in mucopolysaccharidosis type VI (Maroteaux-Lamy) disease. Neuropathol Appl Neurobiol 2005;31:536-44

34. Kwee RM, Kwee TC. Virchow-Robin spaces at MR imaging. Radiographics 2007;27:1071-86

35. Groeschel S, Chong WK, Surtees R, et al. Virchow-Robin spaces on magnetic resonance images: normative data, their dilatation, and a review of the literature. Neuroradiology 2006;48:745-54

36. Valayannopoulos V, Boddaert N, Barbier V, et al. Cognitive and neuroradiological improvement in three patients with attenuated MPS I treated by laronidase. Mol Genet Metab 2010;100:20-23

37. Fan Z, Styner M, Muenzer J, et al. Correlation of automated volumetric analysis of brain MR imaging with cognitive impairment in a natural history study of mucopolysaccharidosis II. AJNR Am J Neuroradiol 2010;31:1319-23

38. Kara S, Sherr EH, Barkovich AJ. Dilated perivascular spaces: an informative radiologic finding in Sanfilippo syndrome type A. Pediatr Neurol 2008;38:363-66

39. Miyazaki T, Masuda N, Waragai M, et al. An adult Japanese Sanfilippo A patient with novel compound heterozygous S347F and D444G mutations in the sulphamidase gene. J Neurol Neurosurg Psychiatry 2002;73:777-78

40. Verhoeven WM, Csepán R, Marcelis CL, et al. Sanfilippo B in an elderly female psychiatric patient: a rare but relevant diagnosis in presenile dementia. Acta Psychiatr Scand 2010;122:162-65

41. Barone R, Nigro F, Triulzi F, et al. Clinical and neuroradiological follow-up in mucopolysaccharidosis type III (Sanfilippo syndrome). Neuropediatrics 1999;30:270-74

42. Lücke T, Das AM, Hartmann H, et al. Developmental outcome in five children with Hurler syndrome after stem cell transplantation: a pilot study. Dev Med Child Neurol 2007;49:693-96

43. Stöckler S, Kleinert R, Ebner F, et al. Mucopolysaccharidosis I and intracranial tumor in a patient with high-pressure hydrocephalus. Pediatr Radiol 1993;23:353-54

44. Vougioukas VI, Berlis A, Kopp MV, et al. Neurosurgical interven- tions in children with Maroteaux-Lamy syndrome: case report and review of the literature. Pediatr Neurosurg 2001;35:35-38

45. Concolino D, Muzzi G, Pisaturo L, et al. Precocious puberty in Sanfilippo IIIA disease: diagnosis and follow-up of two new cases. Eur J Med Genet 2008;51:466-71

46. Ohmi K, Greenberg DS, Rajavel KS, et al. Activated microglia in cortex of mouse models of mucopolysaccharidoses I and IIIB. Proc Natl Acad Sci U S A 2003;100:1902-07

47. Ferrer I, Cusi V, Pineda M, et al. Focal dendritic swellings in Purkinje cells in mucopolysaccharidoses types I, II and III: a Golgi and ultrastructural study. Neuropathol Appl Neurobiol 1988;14:315-23

48. Robertson SJ, Wolpert SM, Runge VM. MR imaging of middle cranial fossa arachnoid cysts: temporal lobe agenesis syndrome revisited. AJNR Am J Neuroradiol 1989;10:1007-10

49. Santamarta D, Aguas J, Ferrer E. The natural history of arachnoid cysts: endoscopic and cine-mode MRI evidence of a slit-valve mechanism. Minim Invasive Neurosurg 1995;38:133-37

50. Ebara S, Kinoshita T, Yuzawa Y, et al. A case of mucopolysaccharidosis IV with lower leg paresis due to thoraco-lumbar kyphoscoliosis. J Clin Neurosci 2003;10:358-61

51. Wilson S, Hashamiyan S, Clarke L, et al. Glycosaminoglycan-mediated loss of cathepsin K collagenolytic activity in MPS I contributes to osteoclast and growth plate abnormalities. Am J Pathol 2009; 175:2053-62

52. Aydin M, Akarsu S, Kabakus N, et al. Mucopolysaccharidosis IIIB, cerebral vasculopathy and recurrent subdural hematoma. Indian Pediatr 2006;43:437-40

53. Chan JW. Pseudotumor cerebri in Morquio type IV syndrome. J Child Neurol 2009;24:514-15

54. Hughes DG, Chadderton RD, Cowie RA, et al. MRI of the brain and craniocervical junction in Morquio's disease. Neuroradiology 1997;39:381-85

55. Vinchon M, Cotten A, Clarisse J, et al. Cervical myelopathy secondary to Hunter syndrome in an adult. AJNR Am J Neuroradiol 1995; 16:1402-03

56. Giussani C, Roux FE, Guerra P, et al. Severely symptomatic craniovertebral junction abnormalities in children: long-term reliability of aggressive management. Pediatr Neurosurg 2009;45:29-36

57. Piccirilli CB, Chadduck WM. Cervical kyphotic myelopathy in a child with Morquio syndrome. Childs Nerv Syst 1996;12:114-16

58. Tandon V, Williamson JB, Cowie RA, et al. Spinal problems in mucopolysaccharidosis I (Hurler syndrome). J Bone Joint Surg $\mathrm{Br}$ 1996;78:938-44

59. Kachur E, Del Maestro R. Mucopolysaccharidoses and spinal cord compression: case report and review of the literature with implications of bone marrow transplantation. Neurosurgery 2000;47:223-28

60. Miebach E, Church H, Cooper A, et al. The craniocervical junction following successful haematopoietic stem cell transplantation for mucopolysaccharidosis type I H (Hurler syndrome). J Inherit Metab Dis 2011;34:755-61. Epub 2011 Mar 17

61. O'Brien DP, Cowie RA, Wraith JE. Cervical decompression in mild mucopolysaccharidosis type II (Hunter syndrome). Childs Nerv Syst 1997;13:87-90

62. Dickerman RD, Colle KO, Bruno CA Jr, et al. Craniovertebral instability with spinal cord compression in a 17-month-old boy with Sly syndrome (mucopolysaccharidosis type VII): a surgical dilemma. Spine (Phila Pa 1976) 2004;29:92-94

63. Mut M, Cila A, Varli K, et al. Multilevel myelopathy in MaroteauxLamy syndrome and review of the literature. Clin Neurol Neurosurg 2005; 107:230-35

64. Pizzutillo PD, Osterkamp JA, Scott CI Jr, et al. Atlantoaxial instability in mucopolysaccharidosis type VII. J Pediatr Orthop 1989;9: $76-78$

65. Hite SH, Krivit W, Haines SJ, et al. Syringomyelia in mucopolysaccharidosis type VI (Maroteaux-Lamy syndrome): imaging findings following bone marrow transplantation. Pediatr Radiol 1997;27: 736-38

66. Samia P, Wieselthaler N, van der Watt GF, et al. Hemiatrophy of the 
spinal cord in a patient with mucopolysaccharidosis type IIIB. J Child Neurol 2010;25:1288-91

67. Davison JE, Hendriksz CJ, Sun Y, et al. Quantitative in vivo brain magnetic resonance spectroscopic monitoring of neurological involvement in mucopolysaccharidosis type II (Hunter Syndrome). J Inherit Metab Dis 2010 Oct 1. [Epub ahead of print].

68. Vedolin L, Schwartz IV, Komlos M, et al. Brain MRI in mucopolysaccharidosis: effect of aging and correlation with biochemical findings. Neurology 2007;69:917-24

69. Segawa F, Kishibayashi J, Kamada K, et al. Diffusion anisotropy in cerebral white matter lesion [in Japanese]. No To Shinkei 1994;46:765-70

70. Sener RN. Diffusion magnetic resonance imaging patterns in metabolic and toxic brain disorders. Acta Radiol 2004;45:561-70
71. Hite SH, Peters C, Krivit W. Correction of odontoid dysplasia following bone marrow transplantation and engraftment (in Hurler syndrome MPS 1H). Pediatr Radiol 2000;30:464-70

72. Wang RY, Cambray-Forker EJ, Ohanian K, et al. Treatment reduces or stabilizes brain imaging abnormalities in patients with MPS I and II. Mol Genet Metab 2009;98:406-11

73. Munoz-Rojas MV, Vieira T, Costa R, et al. Intrathecal enzyme replacement therapy in a patient with mucopolysaccharidosis type I and symptomatic spinal cord compression. Am J Med Genet A 2008;146A:2538-44

74. Muñoz-Rojas MV, Horovitz DD, Jardim LB, et al. Intrathecal administration of recombinant human $\mathrm{N}$-acetylgalactosamine 4-sulfatase to a MPS VI patient with pachymeningitis cervicalis. Mol Genet Metab 2010;99:346-50 